\section{P1.36 COMPARISON OF NUGENT CRITERIA, AMSEL'S CRITERIA AND MODIFIED AMSEL'S CRITERIA FOR DIAGNOSING BACTERIAL VAGINOSIS WITH SPECIAL EMPHASIS ON CONCORDANCE OF SELF AND PHYSICIAN-COLLECTED SAMPLES}

Manju Bala, Zarine Khan, Aradhana Bhargava, Pratima Mittal, Rekha Bharti, Poonam Puri, Niti Khunger. VMMC and Safdarjung Hospital, New Delhi, India

\subsection{6/sextrans-2017-053264.144}

Introduction Bacterial vaginosis (BV) is the most common cause of vaginal discharge worldwide including India. Syndromic diagnosis and management is used across the country for STIs/RTIs. Aims of the study were 1) to evaluate the prevalence of BV based on Nugent's, Amsel's and modified Amsel's criteria 2) to analyse concordance between self- and physician collected swabs for the three different criteria.

Methods Study included 550 females attending STI/RTI clinics with abnormal vaginal discharge during the study period of January 2015 to May 2016. Self-collection of swabs was done by patients after instructions followed by physician collection under speculum examination. The samples were analysed by Nugent's, Amsel's and modified Amsel's criteria for diagnosing BV.

Results Based on Nugent scoring, 79 (14.3\%), 95 (17.3\%), $376(68.4 \%)$ patients were found to be BV positive $(+)$, intermediate and BV negative (-) respectively. However, using Amsel's criteria only $67(12.2 \%)$ patients were observed to be BV+ and $483(87.8 \%)$ were BV-. As modified Amsel's criteria diagnoses BV+ even when only two out of four Amsel's criteria are fulfilled, it could detect $96 \mathrm{BV}+$ cases (17.5\%). All the above results were obtained using the physician-collected samples. Sensitivity for diagnosing BV by self-collected samples using Nugent score, Amsel's criteria and modified Amsel's criteria was $91.1 \%, 98.5 \%$ and $97.9 \%$, while specificity was $100 \%, 99.6 \%$ and $99.6 \%$, positive predictive value $100 \%$, $97.1 \%$ and $97.9 \%$ and negative predictive value was $98.5 \%$, $99.8 \%$ and $99.6 \%$ respectively. High concordance of self-collected samples was established by the Cohen's Kappa value of $0.890,0.975$, and 0.993 for Nugent scoring, Amsel's criteria and Modified Amsel's criteria respectively.

Conclusion Apart from Nugent's scorimg, Amsel's and modified Amsel's criteria were equally consistent methods of diagnosis. Establishing reliability of self-collected samples for diagnosing BV shall pave way for validation of syndromic diagnosis even at peripheral health centres.

\section{P1.37 PREVALENCE AND MOLECULAR ANALYSIS OF MYCOPLASMA GENITALIUM STRAINS ISOLATED FROM PREGNANT WOMEN AT AN ACADEMIC HOSPITAL IN PRETORIA, SOUTH AFRICA}

${ }^{1}$ Le Roux MC, ${ }^{2}$ Mafunise M, ${ }^{1}$ de Villiers BE, ${ }^{1,2}$ Ditsele RMM. ${ }^{1}$ Department of Microbiological Pathology, Sefako Makgatho Health Sciences University; ${ }^{2}$ NHLS Dr George Mukhari Academic Hospital, Pretoria, South Africa

\subsection{6/sextrans-2017-053264.145}

Introduction: M. genitalium is not usually among organisms for routine testing in sexually transmitted infection screening. Treatment follows syndromic guidelines. As M. genitalium cannot be readily cultured, detection of antimicrobial resistance and typing of strains relies on DNA sequence data. It was shown that although there is high intra-strain stability, high levels of sequence variability between clinical isolates are seen which may be associated with antimicrobial resistance.

Methods Endocervical swabs were collected from 100 pregnant women attending a tertiary hospital in Pretoria, South Africa. The specimens were screened for M. genitalium using a commercial real time PCR assay. Genotypic resistance markers for macrolide and fluoroquinolones were determined by sequence analysis of the $\mathrm{V}$-region of the $23 \mathrm{~S}$ rRNA, gyrA, and parC genes. The strains were typed using $\mathrm{mgpB}$ single-nucleotide polymorphism typing (SNP) and MG309 variable number tandem (VNTR) analysis.

Results: M. genitalium was detected in 7 (7.0\%) of specimens of which one positive sample could not be detected with further methods. No resistance associated mutations were seen in the gyrA and parC genes. In 2 isolates the macrolide associated mutation A2059G was seen. SNP typing revealed Sequence Types 1, 2 and 4. Four different types were seen using MG309 VNTR analysis. Typing assigned M. genitalium to 2 major clusters. Genotypic macrolide resistance was found within one of the clusters.

Conclusion: Mycoplasma genitalium is a frequent undiagnosed STD in this population. As azythromycin was included in the national syndromic treatment guidelines in 2015 , it is alarming to already find resistance associated genes.

\section{P1.38 BACTERIAL POPULATIONS DETECTED WITHIN FIRST VOID URINE SAMPLES OF SYMPTOMATIC MALE PATIENTS WITH URETHRITIS}

${ }^{1}$ Marcus Pond, ${ }^{2}$ Josef Wagner, ${ }^{1}$ Ken Laing, ${ }^{1}$ Achyuta Nori, ${ }^{2}$ Julian Parkhill, ${ }^{1}$ Philip Butcher ${ }^{1}$ Tariq Sadiq. 'Institute for Infection and Immunity, St George's University of London, London, UK; ${ }^{2}$ Wellcome Trust Sanger Institute, Hinxton, UK

\subsection{6/sextrans-2017-053264.146}

Introduction Applying bacterial 16S rRNA profiling we investigated whether species in first void urine (FVU) differed between men presenting with urethritis symptoms with and without urethral inflammation.

Methods 443 patients prospectively attending a London sexual health clinic were classified, based on clinical presentation and Gram stain as: symptomatic urethritis (URE); symptomatic non-urethritis (SYM); and asymptomatic (ASP). Residual FVU's were tested for [K1] Chlamydia trachomatis (CT), Neisseria gonorrhoeae (NG), Trichomonas vaginalis (TV) and Mycoplasma genitalium (MG) and subjected to bacterial 16S rRNA profiling (variable region v1-2) Differences in bacterial oligotypes across samples were described using partial least square discriminant analysis tested using permutational multivariate analysis of variance (PERMANOVA). The hypothesis tested was: URE have distinct urinary bacterial 16S rRNA profiles compared to SYM and ASP.

Results 286/443 samples met quality control criteria. Among URE [n=79], SYM [n=83], and ASP [n=124], CT, MG, NG and TV prevalence's were: $12.7 \%$ (95 CI 6.6-22.5), $13.9 \%$ (95CI 7.5-24), $8.9 \%$ (95CI 3.9-7.5), $1.3 \%$ (95CI $0.1-7.8$ ); $2.4 \%$ (95CI $0.4-9.2$ ), 2.4\% (95CI $0.4-9.2$ ), $1.2 \%$ (95CI $0.1-$ $7.5) ; 2.4 \%$ (95CI $0.6-7.4$ ), 5.7\% (95CI 2.5-11.8), NG not detected, $0.8 \%$ (95CI 0-5.1) respectively. Lactobacillus iners was the most abundant oligotype observed in all groups. Streptococcus agalactiae and S. anginosus were highly dominant in ASP along with $S$. mitis, which was relatively increased in both ASP and SYM. A decreased relative abundance of these oligotypes was observed in URE cases. A 
significant difference in oligotype populations was observed between URE and SYM (PERMANOVA test $p=0.0072$, F2.182), but not between SYM and ASP (PERMANOVA test [JW2] $\mathrm{p}=0.0 .1797, \mathrm{~F} 1.601)$.

Conclusion SYM and ASP oligotype populations were dominated by Lactobacillus and Streptococcus species. Microbiota observed in FVU samples of symptomatic patients with microscopy confirmed urethritis (URE) were distinct to those with symptoms but no urethritis on microscopy (SYM).

\section{P1.39 EVALUATION OF A CHEMILUMINESCENCE ASSAY FOR THE DETECTION OF TREPONEMA PALLIDUM ANTIBODIES FOR THE SEROLOGICAL DIAGNOSIS OF SYPHILIS}

${ }^{1}$ Maria Lucia Gallo Vaulet, ${ }^{1} \mathrm{M}$ Mestre, ${ }^{2} \mathrm{D}$ Ori, ${ }^{1} \mathrm{~S}$ Nacher, ${ }^{2} \mathrm{P}$ Baranda, ${ }^{1} \mathrm{M}$ Rodriguez Fermepin. 'Universidad de Buenos Aires, Facultad de Farmacia Y Bioquímica, Depto. Bioquímica Clínica, Buenos Aires, Argentina; ${ }^{2}$ Hospital Naval Cirujano Mayor Dr. Pedro Mallo, Buenos Aires, Argentina

\subsection{6/sextrans-2017-053264.147}

Introduction Syphilis is a sexually transmitted disease caused by Treponema pallidum, which produces 5.6 million new cases worldwide in 2012 and results in significant morbidity and mortality. Despite the availability of diagnostic tests and affordable treatment, the disease remains a global health problem. Detection of non treponemal and treponemal antibodies is the most reliable method for laboratory diagnosis of syphilis. Recently a chemiluminescence microparticle immunoassay (CMIA) has been introduced for the detection of treponemal antibodies and evaluation of its performance against reference methods is needed.

Methods Sera samples were tested with three syphilis serology tests: Venereal Disease Research Laboratory (VDRL) for non treponemal antibodies, fluorescent treponemal antibody absorption (FTA-ABS) test and CMIA. The sensitivity, specificity, positive predictive value (PPV), negative predictive value (NPV) and agreement (Cohen's kappa coefficient) of CMIA was calculated against FTA-ABS (reference group) for the diagnosis of syphilis.

Results Sera samples from 80 patients with suspected syphilis were included in this study. According to serology results: 29 syphilis non reactive sera (VDRL, FTA-ABS and CMIA non reactive), 8 VDRL reactive but FTA-ABS and CMIA non reactive sera and 43 syphilis reactive sera including 10 samples with non reactive VDRL test but FTA-ABS and CMIA reactive (past or treated syphilis) and 33 samples with all reactive tests (ongoing syphilis). General agreement between FTA-ABS and CMIA was $91.2 \%$ with kappa coefficient 0.82 . CMIA clinical sensitivity was $97.7 \%$, clinical specificity was $83.8 \%$, PPV was 87.5\% and NPV 96.9\%.

Conclusion CMIA has the advantages of automation and avoids the subjectivity of FTA-ABS test. According to our results, CMIA has a good agreement with FTA-ABS but has low specificity. Limitations of this test for the diagnosis of syphilis should be taken into account since confirmatory test like FTA-ABS must still be done along with VDRL test and viewed in the context of clinical presentation

\section{P1.40 PRESENCE OF LANGERHANS CELLS AND ACTIVATION OF T LYMPHOCYTES IN DIFFERENTS STAGES OF UTERINE COLO LESIONS ASSOCIATED WITH HUMAN PAPILOMA VIRUS INFECTION}

Maria Odete De Oliveira Carvalho. Fundação Oswaldo Cruz, Rio De Janeiro - RJ, Brazil

\subsection{6/sextrans-2017-053264.148}

Cervical cancer is the fourth most common cancer worldwide, and it is associated with human papillomavirus (HPV) infection. In general, most of cervical lesions regress spontaneously, indicating that HPV infection is not sufficient for inducing carcinogenesis. The immune system has a crucial role in HPV infection control, however there is still much to be investigated. Thus, this study aimed to identify the presence of Langerhans cells $(\mathrm{LC}, \mathrm{CD} 1 \mathrm{a}+)$ and activated $\mathrm{T}$ lymphocytes, induced by HPV-infection. The study enrolled 68 women, aged from 18 to 50 years old. From these, 17 were volunteers without cervical lesion, and 51 presenting HPV-related cervical intraepithelial neoplasia (CIN I-III, $\mathrm{n}=17$ in each group). HPV-DNA was observed in 46 (90.2\%) of $51 \mathrm{CIN}$ volunteers, and all of women from the control group were HPV-negative. We observed an increase of CD45RA+ (naives) cells and CD45RO+ (activated, memory) cells distribution in the epithelium and basal layer of high-grade lesions (CIN II and/or CIN III) when compared with the control group. In relation to LC, our study showed a reduced number of these cells in low-grade lesions in all of investigated areas when compared with the control group, according to pre-malign lesions evolution. For CCL20 analysis, we observed an increased number of these chemokine-expressing cells in the stroma area, in relation to lesions severity. On the other hand, we observed a decrease of these cells in the epithelium. An increase of the distribution of CCR6+ (CCL20 receptor) cells in the basal layer of the epithelium in high-grade lesions (CIN II and III) occurred when compared with the control group. Thus, we concluded that LC and T lymphocytes migrate in direction to the epithelium area, where viral infection occurs, in association with the different cervical lesions degree. However, the inflammatory cells bordering the epithelial areas, focusing the stroma area, indicate that HPV can influence the lesions microenvironment, changing negatively the inflammatory cells recruitment to the site of infection.

\section{P1.41 A SYSTEMATIC REVIEW OF FIELD PERFORMANCE OF DUAL DIAGNOSTIC TESTS FOR HIV AND SYPHILIS: WHAT IS THE MOST APPROPRIATE "GOLD STANDARD" FOR SYPHILIS INFECTION?}

${ }^{1}$ Mary L Kamb, ${ }^{2}$ Harriet Gliddon, ${ }^{3}$ Melanie Taylor. ${ }^{1}$ Centres for Disease Control and Prevention (CDC), Atlanta, Georgia, USA, USA; ${ }^{2}$ University College London, London, England, UK, UK; ${ }^{3}$ World Health Organisation (WHO), Geneva, Switzerland, Switzerland

\subsection{6/sextrans-2017-053264.149}

Introduction Rapid tests (RTs) improve screening and treatment in people without ready access to laboratory testing. Newer RTs linking syphilis and HIV tests on a single device could simplify testing and lower costs. Previous laboratory evaluations of dual HIV/Syphilis RTs suggest high accuracy using trained technicians and characterised sera. However, data from field evaluations would more likely represent "real world" performance. 